\title{
Study of LADRC Controller for Main Steam Temperature System
}

\author{
Yuechao Wang ${ }^{1, a}$, Fengping Pan ${ }^{1}$, Lingling Shi ${ }^{1}$, Zhiqiang Pang ${ }^{1}$, Juanjuan \\ Ren $^{1}$
}

${ }^{1}$ Electric Power Research Institute of Guangdong Grid Company, Guangdong, 510080, China

\author{
ayuechaoster@me.com
}

Keywords: Main steam temperature; LADRC-P cascade control system; robust performance; QPSO; ITAE creteria.

Abstract. Against such characteristics as large delay, nonlinear, time-varying and uncertainty of controlled object, the P-LADRC cascade control system is proposed in this paper. The principle of the LADRC is briefly introduced firstly, and then QPSO algorithm is applied to tune the initial parameters of LADRC-P controller thus achieving better performance of the main steam temperature. In addition, control effect of the new structure and PID cascade control structure is compared. Results show that when using different control strategies for the same load, performance under P-LADRC control is better than that under PID control.

\section{Introduction}

In thermal power plant, boiler superheated steam outlet temperature (main steam temperature) is one of the important parameters affecting the security and efficiency of unit [1]. Considering the main steam temperature control system is a typical large inertia, large delay, nonlinear and time-varying system, the system has a complex structure and difficult to control [2]. Therefore it is very important to control the main steam temperature accurately.

Active disturbance rejection control(ADRC) is an object-model independent control method which was first proposed by Prof. Han in 1998 for rejecting disturbance of a nonlinear system [3]. Literature [4] simplified the ADRC design procedure by considering its 'linear' version and then proposed a linear active disturbance rejection control(LADRC). By using this method the perfect performance can be obtained for multi-variable control systems with small time-delay, but it is difficult to tune parameters of the new controller. The Integral of Time multiply by Absolute Error (ITAE) index is a popular performance criterion used for control system design. The index was proposed by Graham and Lathrop (1953), who derived a set of normalized transfer function coefficients from 2nd-order to 8th-order to minimize the ITAE criterion for a step input[8].

Using quantum-behaved particle swarm optimization(QPSO) algorithm, it is possible to tune a LADRC controller based on the actual transfer function of the plant to optimize the closed-loop performance. In this paper an optimization method is being used to obtain LADRC-P controller parameters. A search of parameters to be optimized lead to select the Integral of Time multiply by Absolute Error (ITAE) index performance criterion, since it can provide controllers with a high load disturbance rejection and minimize the system overshoot while maintain the robustness of the system. Found on the above situation, in this paper a new method for main steam temperature control is presented the LADRC-P cascade control and the framework of the LADRC-P two-loop cascade control system is also constructed to get better control performance.

\section{Design and Tuning of LADRC}

The structure of a second-order LADRC is shown in Fig.1. 


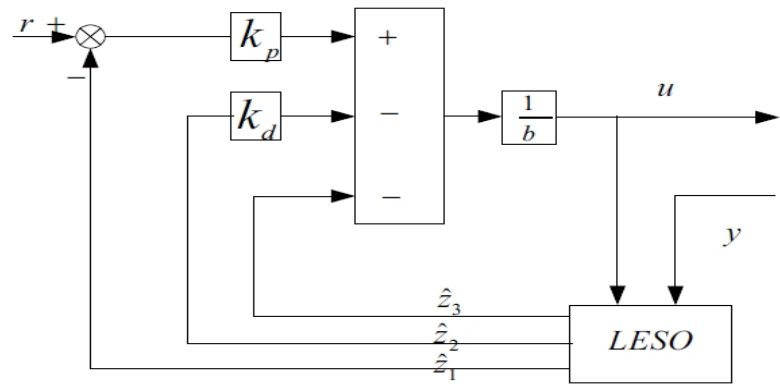

Fig.1. Structure of LADRC

Consider a generalized second-order system given by:

$f(\& y, \imath \ll \omega, t)+b u$

And an extended state observer(ESO) is given by:

$$
z_{1}=y, z_{2}=\notin z_{3}=f(\& y, \imath \& \omega, t)
$$

Assume that $f(\& y, \imath \notin \omega, t)$ is differentiable and let $f(\& y, \imath \leqslant \omega, t)=h$. The above equation(2) is equivalent to:

$$
\left\{\begin{array}{l}
\&=A_{0} z+B_{0} u+E_{0} h \\
y=C_{0} z
\end{array}\right.
$$

where

$$
A_{0}=\left[\begin{array}{lll}
0 & 1 & 0 \\
0 & 0 & 1 \\
0 & 0 & 0
\end{array}\right], B_{0}=\left[\begin{array}{l}
0 \\
b \\
0
\end{array}\right], E_{0}=\left[\begin{array}{l}
0 \\
0 \\
1
\end{array}\right], C_{0}=\left[\begin{array}{lll}
1 & 0 & 0
\end{array}\right]
$$

A standard linear observer for (3) can be written as:

$$
\left\{\begin{array}{l}
\hat{z}=A_{0} \hat{z}+B_{0} u+L_{0}(y-\hat{y}) \\
\hat{y}=C_{0} \hat{z}
\end{array}\right.
$$

where Lo is the observer gain vector

$$
L_{0}=\left[\begin{array}{lll}
\beta_{1} & \beta_{2} & \beta_{3}
\end{array}\right]^{T}
$$

$\hat{z}_{1}, \hat{z}_{2}, \hat{z}_{3}$ can closely track $y, \& f(\&, y, \imath \leqslant \omega, t)$ respectively if the observer gain Lo is chosen properly, thus the generalized disturbance $f$ is available for control.

The control law is chosen as follow:

$$
u=\frac{-\hat{z}_{3}+u_{0}}{b}
$$

ignoring the observer's estimation error, the plant is reduced to a unit gain double integrator:

$$
f(\& y, \ll \alpha \omega, t)-\hat{z}_{3}+u_{0} \approx u_{0}
$$

So, the final system can be easily controlled with a traditional PD(Proportional-Derivative) controller:

$$
u_{0}=K_{p}\left(r-\hat{z}_{1}\right)-K_{d} \hat{z}_{2}
$$

where $r$ is the set point, $\mathrm{Kp}$ and $\mathrm{Kd}$ are PD control parameters. 
For practical reason, the tuning of these two sets of gains are reduced to two tuning parameters: $\omega_{c}$, the controller bandwidth and $\omega_{0}$, the observer bandwidth. For the sake of simplicity and practicality, assume that all observer poles are placed at $-\omega_{0}$, so the parameters can be selected with

$$
\beta_{1}=3 \omega_{0}, \beta_{2}=3 \omega_{0}^{2}, \beta_{3}=\omega_{0}^{3}
$$

If $\hat{z}_{1}, \hat{z}_{2}$ are accurate, then the final controlled system becomes

$$
u_{0}=K_{p}(r-y)-K_{d} £
$$

The closed-loop transfer function from $\mathrm{r}$ to $\mathrm{y}$ is :

$$
G_{c l}=\frac{y(s)}{r(s)}=\frac{K_{p}}{s^{2}+K_{d} s+K_{p}}
$$

By tuning $\mathrm{Kp}$ and $\mathrm{Kd}$, the closed loop can achieve the desired dynamic performance. Similarly, in order to simplify controller parameters, assume that all two of the controller poles are placed at $-\omega_{c}$, then the tuning of $\mathrm{Kp}$ and $\mathrm{Kd}$ is given by:

$$
K_{d}=2 \omega_{c}, K_{p}=\omega_{c}^{2}
$$

The LADRC has the following state-space form:

$$
\left\{\begin{array}{l}
\&=\left(A_{0}-L_{0} C_{0}\right) \hat{z}+B_{0} u+L_{0} y=\left[\begin{array}{ccc}
-3 \omega_{0} & 1 & 0 \\
-3 \omega_{0}^{2} & 0 & 1 \\
-\omega_{0}^{3} & 0 & 0
\end{array}\right] \hat{z}+\left[\begin{array}{cc}
0 & 3 \omega_{0} \\
b & 3 \omega_{0}^{2} \\
0 & \omega_{0}^{3}
\end{array}\right]\left[\begin{array}{l}
u \\
y
\end{array}\right] \\
u=\frac{K_{p}\left(r-\hat{z}_{1}\right)-K_{d} \hat{z}_{2}-\hat{z}_{3}}{b}=\frac{\omega_{c}^{2}\left(r-\hat{z}_{1}\right)-2 \omega_{c} \hat{z}_{2}-\hat{z}_{3}}{b}
\end{array}\right.
$$

\section{Quantum-behaved particle swarm optimization}

A PSO system[5], in which individuals (particles) representing the candidate solutions to the problem at hand fly through the n-dimensional space to find out the optima or sub-optima, got more and more attention according to its explicit mechanism and simple calculation, with the position vector and velocity vector of particle $i$ being represented as $X_{i}(t)=\left(X_{i 1}(t), X_{i 2}(t), \mathrm{L}, X_{i n}(t)\right)$ and $V_{i}(t)=\left(V_{i 1}(t), V_{i 2}(t), \mathrm{L}, V_{i n}(t)\right)$ respectively. For the particle $\mathrm{i}$ and iterative j generation its position is calculated as follow

$$
\begin{aligned}
& v_{i}(t+1)=\omega v_{i}(t)+c_{1} \cdot \operatorname{rand} 1() \cdot\left(\text { pbest }-x_{i}(t)\right)+c_{2} \cdot \operatorname{rand} 2() \cdot\left(\text { pbest }-x_{i}(t)\right) \\
& x_{i}(t+1)=x_{i}(t)+v_{i}(t+1) \quad \text { Where } i=1,2, \mathrm{~L}, N ; t=1,2, \mathrm{~L} L
\end{aligned}
$$

In [5], Sun J. et al proposed that individual particles in a PSO system have quantum behavior. Keeping to the philosophy of PSO, a Delta potential well model of PSO in quantum world is presented, which can depict the probability of the particle's appearing in position $\mathrm{x}$ from probability density function $|\psi(x, t)|^{2}$ not limited to determined trajectory, with the center on point pbest. The particles move according to the following iterative equation ([5], [6]):

$$
X(t+1)=P \pm \beta \cdot \mid \text { mbest }-x(t) \mid \cdot \ln (1 / u)
$$


where

$$
\begin{aligned}
& \text { mbest }=\frac{1}{N} \sum_{i=1}^{N} \text { pbest }_{i} \\
& P=\operatorname{rand}() * \text { pbest }_{i}+(1-\operatorname{rand}()) * \text { gbest }
\end{aligned}
$$

mbest (Mean Best Position) is defined as the mean value of all particles' the best position, rand( and $u$ are random number distributed uniformly on $[0,1]$, respectively. $\beta$, Contraction-Expansion Coefficient, which can be tuned to control the convergence speed of algorithms, is the only parameter in QPSO algorithm.

\section{ITAE index}

ITAE index (the objective function) which is mathematically given by[7]

$$
\text { ITAE }=\int_{0}^{\infty} t|e(t)| d t
$$

where $t$ is the time and $e(t)$ is the error which is calculated as the difference between the set point and the output.

\section{Design of Control Scheme for the Main Steam Temperature System}

In this paper, we adopt an improved cascade control system. The main steam temperature cascade control scheme is shown in Fig.2.

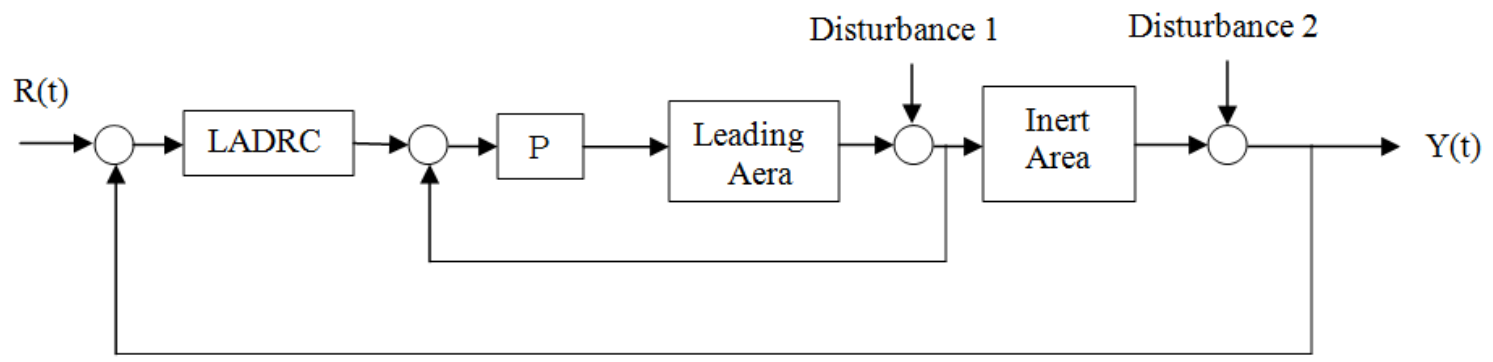

Fig.2. main steam temperature cascade control system

The paper adopts a typical loads of a 600MW supercritical boiler[8], the system structure is shown in Fig.2. And the dynamic characteristics model when the main steam subjects to spray-water disturbance is shown in Table 1:

Table 1. The dynamic characteristics model of main steam temperature

\begin{tabular}{ll}
\hline Temperature of leading area & Temperature of inert area \\
\hline$-1.657 /(1+20 s)^{2}$ & $-1.202 /(1+27.1 s)^{7}$ \\
\hline
\end{tabular}

\section{Test results}

In LADRC-P cascade control system, LADRC parameters are optimazed by QPSO to minimize the ITAE. Parameter Kp in secondary loop remains the same in two control schemes which is chosen as:

\begin{tabular}{|c|c|c|c|c|c|c|}
\hline Parameter & $\begin{array}{l}\text { Population } \\
\text { size }\end{array}$ & Iterations & Centers & C & Width $B$ & $\begin{array}{l}\text { Weight } \\
W\end{array}$ \\
\hline Value & 20 & 2000 & {$[-3,3]$} & & {$[0.1,3]$} & {$[-1,1]$} \\
\hline
\end{tabular}
$K_{p}=-10$. The parameters of the QPSO algorithm are set as Table 2.

Table 2. Execution parameters for QPSO 
According to the tuning method mentioned above, the parameters of the 2nd-order LADRC are given in Table 3:

Table 3. Parameters of LADRC

\begin{tabular}{lllll}
\hline Parameter & $b$ & $\omega_{0}$ & $k_{p}$ & $k_{d}$ \\
\hline Value & 0.001 & 0.05 & 0.0005 & 0.008 \\
\hline
\end{tabular}

Meanwhile, PID cascade control for main steam temperature system is also simulated in this paper as comparison. PID parameters are tuned at same load, which are chosen as Table 4 :

Table 4. Parameters of PID

\begin{tabular}{llll}
\hline Parameter & $k_{p}$ & $k_{i}$ & $k_{d}$ \\
\hline Value & -0.3148 & -0.00717 & 30.935 \\
\hline
\end{tabular}

LADRC-P cascade control is compared with PID cascade control at the typical load by simulation approach is shown in Fig.3

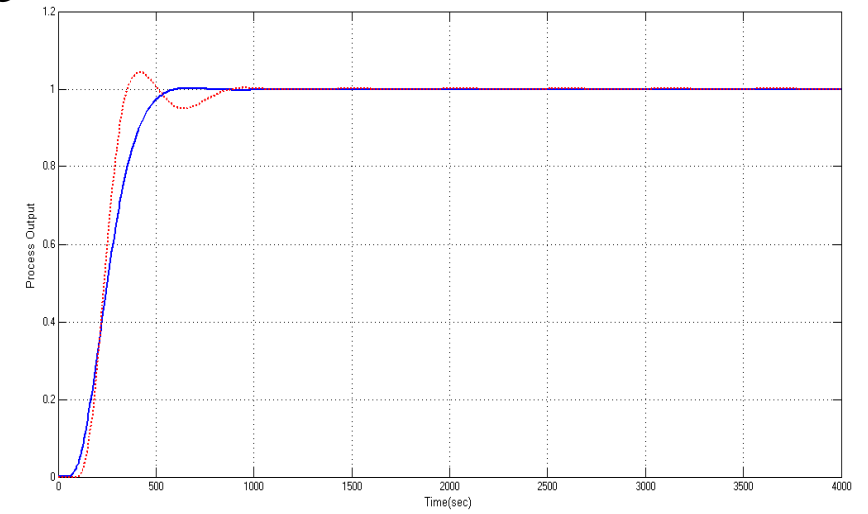

Fig.3.Responses for the typical load (blue: LADRC-P, red: PID-Smith)

The LADRC-P cascade control is compared with PID cascade control by simulation approach. The step responses for the closed-loop system are shown in Fig.3. From Fig.3, it can be concluded that the performance of LADRC-P cascade control system is superior to the conventional PID cascade control system, and the former strategy can overcome the contradictions of rapidity and stability.

There will be an obvious change of the dynamic characteristics of the main steam temperature along with the change of load and working condition. Remain controller parameters unchanged, the main steam temperature object change into the following form due to the load change of the system, as shown in Table 5.

Table 5. The changed model of main steam temperature

\begin{tabular}{ll}
\hline Temperature of leading area & Temperature of inert area \\
\hline$-0.815 /(1+18 s)^{2}$ & $-1.276 /(1+18.4 s)^{6}$ \\
\hline
\end{tabular}




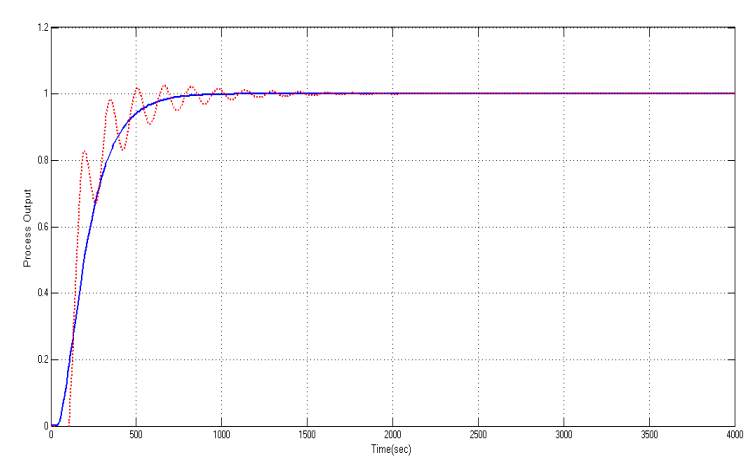

Fig.4.Responses for $20 \%$ load changed

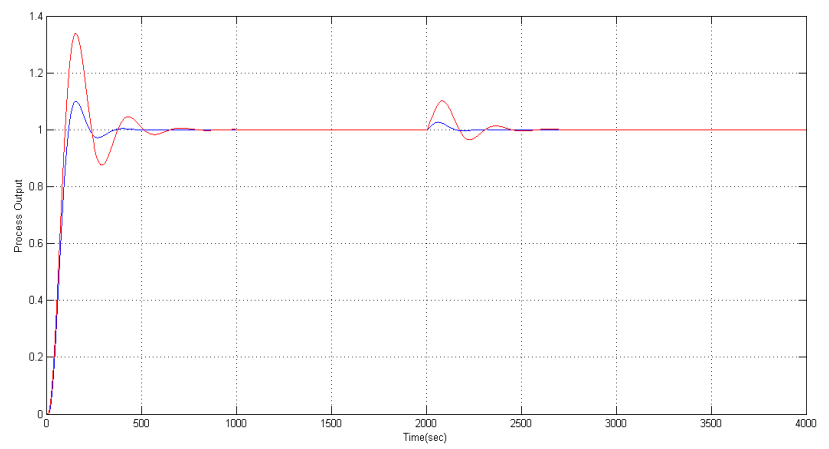

Fig.5.Responses to a steam temperature disturbance (blue: LADRC-P, red: PID-Smith)

The algorithm and controller parameters remain the same, the responses to main steam temperature disturbance for the closed-loop system are shown in Fig.4. It can be seen that the transient time and overshoot of the novel control strategy is smaller than the conventional one, so the proposed method can achieve better control performance and robustness.

In the simulation, a load step disturbance occurs at $\mathrm{t}=2000 \mathrm{~s}$, the response curves are shown in Fig.5. Obviously the proposed method has a strong capacity of resisting disturbance and achieves good control quality.

\section{Conclusion}

A novel cascade control scheme was proposed for main steam temperature of a 600MW supercritical boiler was studied. The LADRC-P controllers are used to further reduce the disturbances and model uncertainties. The parameters of PID controller are adjusted by ITAE index, and the QPSO algorithm is put forward to optimize the initial parameters of LADRC. Simulation results show that the novel control structure can achieve better disturbance rejection performance and robustness and is worth to implement in practice.

\section{References}

[1] Y. Z. Yu, Z. B. Zhang, H. C. Xu, D. G. Chen, G. J. Dong, B. Shen, S. Liu. "Investigation for 600 MW Supercritical Boiler's Main Steam Temperature Control," Proceedings of the 2010 International Conference on Electrical and Control Engineering (ICECE 2010), p 3848-51, 2010

[2] J. T. Zhang, W. Wang, F. Cao. "Application of an intelligent control method in 300MW unit main steam temperature control system," Chinese Society for Electrical Engineering, vol. 19, no. 3, (1999), pp. 6-10.

[3] J. Han. "Active disturbance rejection controller and its applications (in Chinese)," Control and Decision, 1998,13(1):19-23.

[4] J. Gao. "Active disturbance rejection control: A paradigm shift in feedback control system design," American Control Conference, 2006. IEEE, 2006: 7 pp.2399-2405.

[5] Sun, J., Feng, B., Xu, W.B.: Particle Swarm Optimization with Particle Having Quantum Behavior. Proceedings 2004 Congress on Evolutionary Computation, Piscataway, NJ (2004) 325-331.

[6] Sun, J., Xu, W.B., Feng, B.: A Global Search Strategy of Quantum-behaved Particle Swarm Optimization. Proceedings of 2004 IEEE Conference on Cybernetics and Intelligent Systems. Singapore (2004) 111-116.

[7] Awouda A E A, Mamat R B. Refine PID tuning rule using ITAE criteria[C]// The, International Conference on Computer and Automation Engineering. IEEE, 2010:171-176. 
[8] Q. Z. Li, Y. G. Niu, B. L. Lu and Z. C. Lan, "Performance evaluation of thermal power plant superheated steam temperature control system," Electric Power Science and Engineering, vol. 27, no. 7, (2011), pp. 45-49. 